\title{
Computational Alanine Scanning Mutagenesis: Characterizing the hotspots of ILK-Ankyrin Repeat and PINCH1 Complex
}

\author{
Vertika Gautam $^{1 *}$, Nadia Hanim Sabri ${ }^{1}$, Wei Lim Chong ${ }^{1}$, Sharifuddin M. Zain ${ }^{1}$, Noorsaadah Abd. Rahman ${ }^{1}$, \\ Vannajan Sanghiran Lee ${ }^{1,2}$, Anand Gaurav ${ }^{3}$ \\ ${ }^{I}$ Department of Chemistry, Faculty of Science, University of Malaya 50603, Kuala Lumpur Malaysia. \\ ${ }^{2}$ Computational Simulation Modelling Laboratory (CSML), Department of Chemistry and Center of Excellence for Innovation in Chemistry \\ and Materials Science Research Center, Faculty of Science, Chiang Mai University, Chiang Mai 50200,Thailand. \\ ${ }^{3}$ Faculty of Pharmaceutical Sciences UCSI University, Kuala Lumpur, 56000, Malaysia.
}

Received: 3 September, 2014 / Accepted: 30 November 2014

\begin{abstract}
:
From the last two decades, computational alanine scanning mutagenesis (cASM) have been successively applied to a variety of protein complexes to study the energetics and structural characteristics of hot spot residues at protein interface. The cASM combines a continuum approach to model solvent interactions with a MM-based approach to atomistically model protein-protein interactions. In the present study, this methodology was used to study the hotspot residues involved in the binding complex of Integrin linked kinase containing Ankyrin repeats with PINCH1 which have profound effect in cell migration, spreading and signalling. Molecular dynamic simulations using a continuum solvent approach (MM-PB/GBSA) were used in one of our previous study and later alanine scanning with post processing protocol was done as a downstream analysis to predict accurately the differences in binding free energies in solution between wild-type and alanine mutated complexes $\left(\Delta \Delta \mathrm{G}_{\text {bind }}\right)$.
\end{abstract}

Key words: AMBER, ankyrin, cASM, hot-spot, ILK, MM-PBSA, PINCH, protein-protein interactions

\section{Introduction}

Dengue Protein-protein interactions play crucial roles in regulating biological processes, cellular and signal pathway. One of the key features of these proteinprotein interfaces is their sensitivity to mutations, means that if we mutate a key interface residue by a residue alanine, there will be a significant variation in the protein-protein complex binding or association free energy. It has been defined in the literature that if the increase in the binding free energy is above $4 \mathrm{kcal} / \mathrm{mol}$, then the mutated residue is extremely important and it is called a hot spot; if the energy increase upon mutation is between 2 and $4 \mathrm{kcal} / \mathrm{mol}$, then this residue is relatively important for the protein-protein association and it is denominated a warm spot, and finally, if the mutations originate a binding free energy variation below 2 $\mathrm{kcal} / \mathrm{mol}$, then the residue is not particularly relevant for the interaction and it is termed a null spot [1].

The Ankyrin repeat (AR) protein can be used as a versatile scaffold for protein-protein interactions. It has been found that in the Integrin Linked kinase pathway there is a hetero-trimeric (IPP) complex between ILK whose N-Terminal contains 5 ANK repeats, PINCH1 a focal adhesion protein and an obligate partener of ILK containing 5 LIM domains, and $\alpha$-parvin an adapter proteins [2]. ILK PINCH1 association is critical to integrin-mediated cell adhesion, migration, spreading, and signalling. The recent findings of increased levels of ILK in various cancers, and that inhibition of ILK expression and activity is antitumorigenic, makes ILK an attractive target for cancer therapeutics [3]. cASM methods are applied extensively for PPI for this purpose [4]. In the present study cASM is particularly used to identify the hotspots which are involved in AR-ILK and PINCH1 binding.

\section{Experimental}

The initial structure of the ILK Ankyrin repeat domain bound to the PINCH1 LIM1 domain complex was taken from the $\mathrm{x}$-ray crystallography structure with PDB code of 3F6Q [5]. The consensus of Ankyrin repeat is shown in Figure 1.

For cASM, Post-processing protocol is a method used to generate a trajectory of an alanine mutant. In this case, only the MD trajectory of the native (wildtype) complex is run. MD simulations at the molecular mechanics level were employed using ff12SB force field as implemented in the AMBER12 suite of programmes.

\footnotetext{
*Corresponding author: Vertika Gautam,

E-mail:vartikapisces@gmail.com
} 


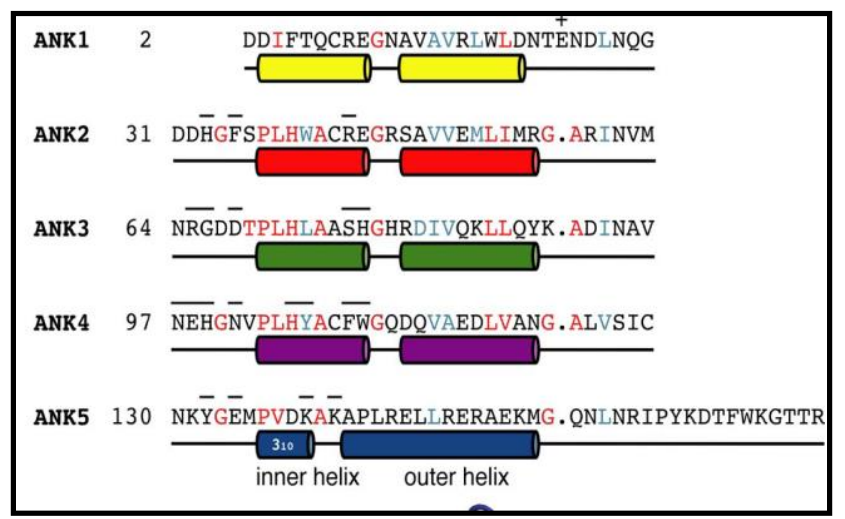

Figure 1. Alignment of the ILK ANK repeats.

Barred Residues: Interacts with PINCH; Red Residues: Highly conserved; Cyan Residues: Reasonably conserved.

The ankyrin-PINCH complex were solvated in a cubic box of TIP3P water extending at $10 \AA$ in each direction from the solute with $9 \mathrm{Na}^{+}$ions added as neutralizing counterions and the cut-off distance was kept to $20 \AA$ to compute the nonbonded interactions. All simulations were performed under periodic boundary conditions, and long-range electrostatics was treated by using the particle-mesh-Ewald method. Energy minimization and Molecular dynamics (MD) simulations were performed using PMEMD.CUDA from AMBER12 on graphical processors (GPUs) Quadro 2000D produced by NVIDIA.

The ff12SB force field parameters implemented in the AMBER programme were applied to describe the molecular characteristics of the complex. Initially, the temperature of each system was increased gradually from 0 to $310 \mathrm{~K}$ over a period of $60 \mathrm{ps}$ of NVT dynamics. This was followed by 300 ps of NPT equilibration at $310.15 \mathrm{~K}$ and $1 \mathrm{~atm}$ pressure and then $10,000 \mathrm{ps}$ of NPTMD simulation was performed for properties collection. The structural properties and intermolecular interactions of the ankyrin-kinase were analyzed from the MD trajectories of $10 \mathrm{~ns}$. The binding free energy of the complex was calculated based on the MM-PBSA/GBSA protocol. The 2500 snapshots were extracted from each range and the grid size for the PB calculations in MMPBSA was $0.5 \AA$.

After the simulation, individual structure snapshots from the 2 ns trajectory were mutated to alanine at the desired residue, by truncating back to the $\mathrm{C}_{\beta}$ atom. The additional two (for $\mathrm{V} / \mathrm{I} / \mathrm{T}$ residues) or one (for all other residues) hydrogen atoms of the alanine methyl side chain are added at the standard $\mathrm{C}-\mathrm{H}$ bond length along the vectors of the previous $\mathrm{C}_{\& \mathrm{bgr} ;}-\mathrm{C}_{\gamma}$ bond or bonds. Thus, a trajectory is generated for the mutant complex in which the coordinates of every residue other than the alanine mutant are identical to those in the native trajectory $(2 \mathrm{~ns})$.

\section{Results and Discussion}

Dynamic conformational changes of complex AR $3 F 6 Q$

The structural properties and dynamic conformational change of AR ILK and PINCH1 were examined based on the Root Mean Squared Deviations (RMSD) of all $\mathrm{C}_{\alpha}$-atoms of AR 3F6Q (Fig. 2). Most of the complexes are simulated in presence of water in order to reveal the properties after the simulation particularly the stability of the complexes. The simulated complex should not deviate much from the initial structure. Root Mean Square Deviation (RMSD) is very often used in examining the structural properties and dynamic conformational change of all $\mathrm{C} \alpha$ of the simulated complex. The RMSD plot for 3F6Q system was shown in Figure 2. Steady oscillations of RMSD plot collected throughout the $10 \mathrm{~ns}$ of simulation time. Fluctuation occurs within the acceptable range of $1-3 \AA$. The complex was found to be stable at 2 ns and 9 ns. It also implied that the equilibrium has obtained.

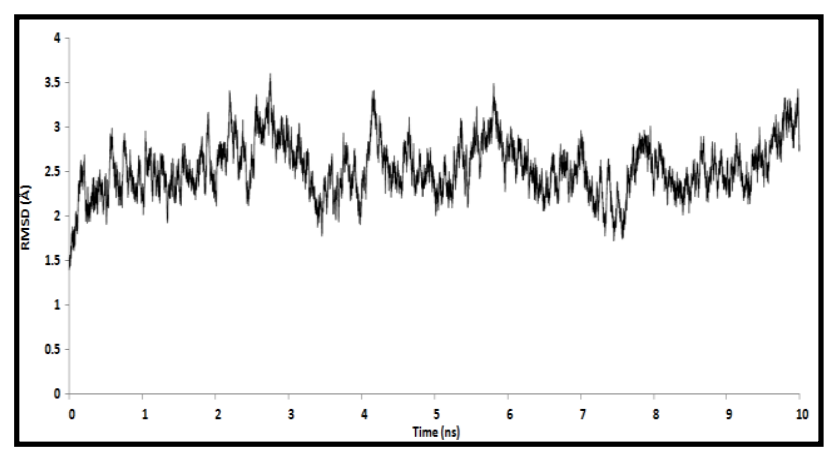

Figure 2. Root Mean Squared Deviations (RMSD) of all $\mathrm{C} \alpha$ atoms of AR ILK-PINCH1 as a function of simulation time.

\section{Important amino acid residues involving in binding of} the complex

On the basis of the RMSD curve it was found that the complex was stable at 2 ns and 9ns trajectories. Further the complex was subjected for the decomposed energy evaluation for the above trajectories and most of the important binding residues with negative energies were found to be in agreement with the experimental data as shown in Figure 1. As reported by Chiswell et al., the residues belonging to ANK4 is the repeat most intrinsic to the ILK-PINCH1 interaction. Four residues from the ANK4 inner helix (Trp110, Phe109, Tyr106, and His105) and 4 residues from the ANK4 hairpin (Asn97, Glu98, His99, and Asn101) contribute to the interaction. The residues with negative binding energy are reported in Table 1. Most of the residues are vis-á-vis with the experimental data.

\section{Computational Alanine Scanning for Hot-Spots}

Post processing protocol was employed for cASM. The binding free energy difference between an alanine mutant and the wild type complex is defined by the Eq.1:

$\Delta \Delta \mathrm{G}_{\text {binding }}=\Delta \mathrm{G}_{\text {binding-mutant }}-\Delta \mathrm{G}_{\text {binding-wildtype }}$ 
For this purpose residues which are involved in ILKPINCH interaction were subjected to cASM. Based on the criterion of $\Delta \Delta \mathrm{G}$, defined by Morriera et al., residues were grouped into Hot, warm and null spots shown in Table 2.

Table 1. Decomposed energy for selected residues (2ns)

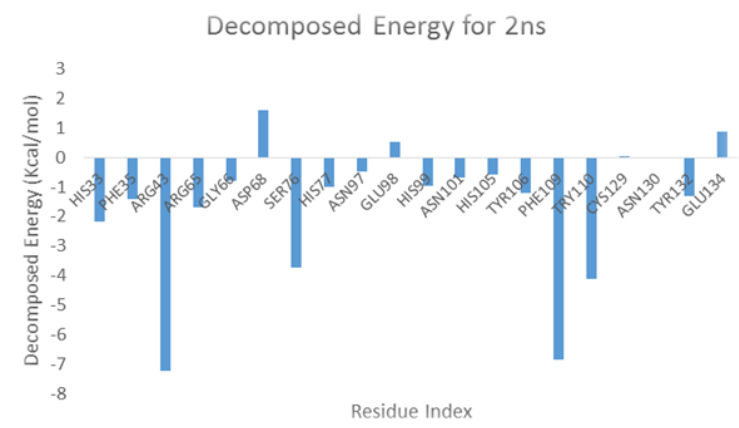

Table 2. Computational Alanine scanning Mutation analysis for the selected AR-ILK residues

\begin{tabular}{lcccl}
\hline Residues & $\boldsymbol{\Delta G}_{\mathbf{m u t a n t}}$ & $\boldsymbol{\Delta} \mathbf{G}_{\mathbf{w t}}$ & $\boldsymbol{\Delta \Delta \mathbf { G }}$ & Remark \\
\hline His33Ala & -41.21 & -44.3 & 3.09 & Warm Spot \\
Arg43Ala & -32.54 & -44.3 & 11.76 & Hot Spot \\
Asp68Ala & -42.10 & -44.3 & 2.20 & Warm Spot \\
Ser76Ala & -38.72 & -44.3 & 5.58 & Hot Spot \\
His77Ala & -42.44 & -44.3 & 1.86 & Null Spot \\
Glu98Ala & -45.29 & -44.3 & -0.99 & Null Spot \\
His99Ala & -40.59 & -44.3 & 3.71 & Warm Spot \\
Asn101Ala & -42.05 & -44.3 & 2.25 & Warm Spot \\
Tyr106Ala & -39.79 & -44.3 & 4.51 & Hot Spot \\
Trp110Ala & -34.66 & -44.3 & 9.64 & Hot Spot \\
Tyr132Ala & -42.18 & -44.3 & 2.12 & Warm Spot \\
Glu134Ala & -45.23 & -44.3 & -0.93 & Null Spot \\
\hline
\end{tabular}

\section{Conclusions}

In the present study, cASM (post processing protocol) was used as the downstream analysis of long time scale MD simulation, to identify the hot-spots of AR ILK-
PINCH1 interaction. The dynamic conformational changes of the AR complex, 3F6Q, were observed by RMSD of all the $\mathrm{C}_{\alpha}$ atoms in which steady oscillations indicate that the equilibrium has been obtained. Also the complex was stable at $2 \mathrm{~ns}$ and $9 \mathrm{~ns}$ trajectories. Residues intrinsic to ILK-PINCH1 interaction were analyzed through decomposed energy studies. A post processing protocol for alanine scanning mutation was used to identify the hot spot. The results indicate that there are residues like ARG42, SER75, TYR105 and TRP110 which are Hot-spots; suggesting, these residues can be further explored with other available amino acids for a better binding of ILK-AR with PINCH1. Moreover, most of the results corroborates with the findings of Chiswell et al,. The findings of the present studies can be treated as the computational validation of the experimental findings.

\section{Acknowledgement}

This research is supported by University Malaya Research Grant under Computation and Informatics $(\mathrm{C}+\mathrm{i})$ Research Cluster / High Performance Scientific Computing Program (UMRG Project no. RP001C13ICT), Biomolecular modelling and stimulating for Protein Design (RP020C-14AFR) Fundamental Research Grant Scheme (FP013-2013A).

\section{References}

[1] I.S. Moreira, P.A. Fernandes, and M.J. Ramos, Hot Spots- a review of the protein-protein interface determinant amino-acid residues, Proteins., 68(23), 2007, 8136-8147.

[2] K.R. Legate, E. Montanez, O. Kudlacek, and R. Fassler, ILK, PINCH and parvin: The tIPP of integrin signalling, Nat. Rev. Mol. Cell Biol., 7, 2006, 20-31.

[3] G. Hannigan, A.A. Troussard, and S. Dedhar, Integrin-linked kinase: A cancer therapeutictarget unique among its ILK, Nat. Rev. Cancer., 2005, 5:51-63.

[4] I. Massova and P.A. Kollman, Computational alanine scanning to probe protein-protein interactions: a novel approach to evaluate binding free energies, J. Am. Chem. Soc., 121(36), 1999, 8133-8143.

[5] P. Brian and R. Chiswell, The structural basis of integrinlinked kinase-PINCH interactions, PNAS., 105(52), 2008, 20677-20682. 